\title{
ON UNIVALENT FUNCTIONS CONVEX IN ONE DIRECTION
}

\author{
A. W. GOODMAN ${ }^{1}$ AND E. B. SAFF $^{2}$
}

\begin{abstract}
Let $f(z)=z+\sum_{2}^{\infty} a_{k} z^{k}$ be analytic and univalent in the unit disk $E:|z|<1$ and map the disk onto a domain which is convex in the direction of the imaginary axis. We show by example that for $\sqrt{2}-1<r<$ 1 , the function $f(z)$ need not map the disk $|z|<r$ onto a domain convex in the direction of the imaginary axis. We also find the largest domain contained in $f(E)$ for every normalized $f(z)$ that maps $E$ onto a domain convex in the direction of the imaginary axis.
\end{abstract}

1. Introduction. A domain $\mathscr{D}$ is said to be convex in the direction of the imaginary axis if for every vertical line $\mathcal{L}$, the set $\mathcal{L} \cap \mathscr{D}$ is either $\mathcal{L}$, an open interval, or the empty set. Any domain that is convex in one direction can be rotated so that it is convex in the direction of the imaginary axis. We let $C D$ denote the set of normalized functions

$$
f(z)=z+\sum_{2}^{\infty} a_{k} z^{k}
$$

that are regular and univalent in $E:|z|<1$ and for which $f(E)$ is a domain that is convex in the direction of the imaginary axis. A representation formula for a certain subclass of functions in $C D$ was given by Robertson in [3]. Recently Royster and Ziegler [4], using the results of Hengartner and Schober [1], showed that Robertson's representation formula does in fact apply to the whole class $C D$.

If $f(z)$ belongs to some set $\mathscr{F}$ of functions defined by a geometric property, it often occurs that the function $f(r z) / r$ is in the same set $\mathscr{F}$ for every $r$ in $(0,1)$. This is certainly true for convex functions, starlike functions, typically real functions, and many other sets. Thus it was a surprise, when Hengartner and Schober [2] proved that the set $C D$ does not have this property. If $\mathscr{D}$ is a domain that is convex in the direction of the imaginary axis, it may happen that the domains bounded by the level curves do not share this property.

Let $R_{C D}$ be the radius of convexity in the direction of the imaginary axis for

Received by the editors March 15, 1978.

AMS (MOS) subject classifications (1970). Primary 30A32.

1 Supported in part by the Samuel Neaman Fund, Special Year in Complex Analysis, Technion, I.I.T., Haifa, Israel, 1975/1976.

${ }^{2}$ Research supported in part by the Air Force Office of Scientific Research under Grant AFOSR-74-2688. 
the set $C D$. Thus $R_{C D}$ is the largest $\rho$ with the property that $f(r z) / r \in C D$ for every $f(z) \in C D$ and every $r$ in $(0, \rho)$. Hengartner and Schober proved that $R_{C D}<1$, but their method was qualitative and they gave no numerical bounds. Here we prove by an example that $R_{C D} \leqslant \sqrt{2}-1 \approx 0.4142$. Since any convex function is convex in every direction, and the radius of convexity for the set $S$ of univalent functions is $2-\sqrt{3} \approx 0.2679$, this gives a trivial lower bound. Thus $2-\sqrt{3} \leqslant R_{C D} \leqslant \sqrt{2}-1$. It seems reasonable to conjecture that $R_{C D}=\sqrt{2}-1$.

2. The example function. Let

$$
F(z)=\frac{z-A z^{2}}{(1-B z)^{2}}, \quad A=e^{2 i \alpha} \cos \alpha, \quad B=e^{i \alpha},
$$

and $\alpha$ is real. For convenience we impose the additional restriction that $0<\alpha<\pi$.

LEMMA 1. For each fixed $\alpha, 0<\alpha<\pi$, the function $F(z)$ is in CD. Further $F(E)$ is the set obtained by deleting from the complex plane the points on the vertical half-line that starts at the point

$$
s_{0}=F\left(e^{-3 i \alpha}\right)=-\frac{\cos \alpha}{2}+i\left(\frac{\sin \alpha}{2}-\frac{1}{4 \sin \alpha}\right)=\frac{e^{-2 i(\alpha-3 \pi / 4)}}{4 \sin \alpha}
$$

and extends upward to $-(\cos \alpha) / 2+i \infty$.

Proof. Since $F^{\prime}(z)=[1+(B-2 A) z] /(1-B z)^{3}$, the only critical point is the simple one at $z_{0}=e^{-3 i \alpha}$ and an easy computation gives $F\left(z_{0}\right)=s_{0}$. For the boundary of $E$ we find that

$$
F\left(e^{i \theta}\right)=-\frac{\cos \alpha}{2}+i \frac{\sin \alpha+\sin (\alpha+\theta) \cos \alpha}{2(1-\cos (\alpha+\theta))} .
$$

Hence the boundary goes into a subset of the vertical line $\operatorname{Re} w=$ $-(\cos \alpha) / 2$. The existence of a simple critical point at $z_{0}=e^{-3 i \alpha}$ shows that the subset is a doubly covered half-line, and the direction is established by a consideration of $F\left(e^{i \theta}\right)$ when $\theta$ is near $-\alpha$. The argument principle shows that $F(z)$ is univalent in $E$. Hence $F(z) \in C D$.

We now examine the behaviour of $\operatorname{Re} F(z)$ on the circle $|z|=r$. Setting

$$
U^{\prime}(r, \theta) \equiv \frac{\partial}{\partial \theta} \operatorname{Re} F\left(r e^{i \theta}\right)=\operatorname{Re}\left[i z F^{\prime}(z)\right]=-\operatorname{Im}\left[z F^{\prime}(z)\right],
$$

we see that if $U^{\prime}(r, \theta)$ has four changes of sign in the interval $[0,2 \pi)$ then for fixed $r, F(r z) / r$ is not in $C D$.

Since we are concerned with the shape of the level curves of $F(z)$ we may replace $F(z)$ by $G(z) \equiv F\left(e^{-i \alpha} z\right)$. A moderate computation gives, for $z=$ $r e^{i \theta}$, 


$$
\begin{aligned}
-\mid 1- & \left.z\right|^{6} \frac{\partial}{\partial \theta} \operatorname{Re} G\left(r e^{i \theta}\right)=|1-z|^{6} \operatorname{Im}\left[z G^{\prime}(z)\right] \\
& =\operatorname{Im}\left\{\left(e^{-i \theta} z-e^{i \alpha} z^{2}\right)(1-\bar{z})^{3}\right\} \\
& =r\left(1-r^{2}\right)\left[\left(1+r^{2}\right) \sin (\theta-\alpha)+r(3 \sin \alpha-\sin (2 \theta+\alpha))\right] .
\end{aligned}
$$

From equation (6) it follows that the number of changes of sign of $U^{\prime}(r, \theta)$ on the circle $|z|=r$ is equal to the number of changes of sign of

$$
Q(\theta) \equiv\left(1+r^{2}\right) \sin (\theta-\alpha)+r[3 \sin \alpha-\sin (2 \theta+\alpha)] .
$$

3. An upper bound for $R_{C D}$. In this section we show that for each $r$ $>\sqrt{2}-1$, there is an $\alpha$ such that $Q(\theta)$ has 4 changes of sign for $\theta$ in $[0,2 \pi)$. We recall that $0<\alpha<\pi$. Hence $Q(0)=-(1-r)^{2} \sin \alpha<0$ for all $r<1$. Further $Q(\pi)=(1+r)^{2} \sin \alpha>0$. Next

$$
Q(3 \pi / 2)=-\left(1+r^{2}\right) \cos \alpha+4 r \sin \alpha,
$$

and so $Q(3 \pi / 2)<0$ whenever

$$
\tan \alpha<\left(1+r^{2}\right) / 4 r,
$$

and $0<\alpha<\pi / 2$. We will see that for each fixed $r>\sqrt{2}-1$, we can select an $\varepsilon, 0<\varepsilon<\pi / 2$, and an $\alpha<\pi / 4$ such that (9) is true and $Q(3 \pi / 2+\varepsilon)>$ 0 . Then $Q(\theta)$ will have 4 changes of sign for $\theta$ in $[0,2 \pi)$. First

$$
\begin{aligned}
h(\varepsilon) \equiv & \frac{Q(3 \pi / 2+\varepsilon)}{r \cos \alpha}=-\frac{1+r^{2}}{r}(\cos \varepsilon+\tan \alpha \sin \varepsilon) \\
& +3 \tan \alpha+\sin 2 \varepsilon+\tan \alpha \cos 2 \varepsilon .
\end{aligned}
$$

We expand $h(\varepsilon)$ in a Taylor series about $\varepsilon=0$ and obtain

$$
\begin{aligned}
h(\varepsilon) & =h(0)+h^{\prime}(0) \varepsilon+h^{\prime \prime}(0) \varepsilon^{2} / 2+M \varepsilon^{3} \\
& \equiv-\delta+\lambda \varepsilon+\delta \varepsilon^{2} / 2+M \varepsilon^{3},
\end{aligned}
$$

where

$$
\delta \equiv \frac{1+r^{2}}{r}-4 \tan \alpha, \quad \lambda \equiv 2-\frac{1+r^{2}}{r} \tan \alpha,
$$

and $M=M(r, \alpha)$ is a bounded term for $0<\alpha<\pi / 4$ and $\sqrt{2}-1<r<1$. Notice that (12) implies

$$
\lambda=\tau+\frac{1+r^{2}}{4 r} \delta, \quad \text { where } \tau \equiv 2-\left(1+r^{2}\right)^{2} / 4 r^{2} .
$$

Suppose now that $r$ is fixed and $1>r>\sqrt{2}-1$. With this $r$ we have $\tau>0$. In order for inequality (9) to hold we will only consider angles $\alpha$ such that $\delta>0$. As $r>\sqrt{2}-1$, the inequality

$$
0<\delta=1+r^{2} / r-4 \tan \alpha<2 \sqrt{2}-4 \tan \alpha
$$

in particular implies that $0<\alpha<\pi / 4$. Next we show that by choosing 
$\delta>0$ sufficiently small (i.e., by proper selection of $\alpha$ ) we have $h\left(\varepsilon_{0}\right)>0$, for $\varepsilon_{0} \equiv 2 \delta / \tau\left(0<\varepsilon_{0}<\pi / 2\right)$. Indeed (11) and (13) yield

$$
\begin{aligned}
\frac{h\left(\varepsilon_{0}\right)}{\varepsilon_{0}^{3}} & =\frac{-\delta}{\varepsilon_{0}^{3}}+\left(\tau+\frac{1+r^{2}}{4 r} \delta\right) \frac{1}{\varepsilon_{0}^{2}}+\frac{\delta}{2 \varepsilon_{0}}+M \\
& =\frac{-\tau^{3}}{8 \delta^{2}}+\frac{\tau^{3}}{4 \delta^{2}}+\left(\frac{1+r^{2}}{16 r}\right) \frac{\tau^{2}}{\delta}+\frac{\tau}{4}+M,
\end{aligned}
$$

and since $\tau>0$, we obtain $h\left(\varepsilon_{0}\right) / \varepsilon_{0}^{3}>\tau^{3} / 8 \delta^{2}+M$. Clearly as $\delta \rightarrow 0^{+}$, the right side of this last inequality goes to $+\infty$. Hence $h\left(\varepsilon_{0}\right)>0$ for $\delta>0$ sufficiently small. But from (10) this means that for suitable $\alpha$ the trigonometric polynomial $Q(\theta)$ has 4 changes of sign on $[0,2 \pi)$. We have proved that $R_{C D} \leqslant \sqrt{2}-1$.

4. The domain covered by $f(E)$. Suppose that $f(z) \in C D$ and omits the point $a+b i$. Then it must omit every point on one of the two vertical half-lines $a+(b+s) i$, with $s \geqslant 0$ or $s \leqslant 0$. In the latter case we consider $\overline{f(\bar{z})}$ in place of $f(z)$. Thus we may assume that $f(z)$ omits the vertical line $a+(B+s) i, s \geqslant 0$, where $B=b$ or $-b$ and $a+B i$ is the endpoint of the omitted half-line. If $a=0$, then we must have $B>0$.

Let $G(w)$ be the inverse of $F(z)$ defined by (2). It is intuitively clear that we can select $t>0$ and $\alpha$ such that the composite function $B(z) \equiv G(t f(z))$ satisfies the conditions of Schwarz's lemma.

To prove this assertion we must find $t$ and $\alpha$ so that the endpoints coincide for the omitted rays of $t f(z)$ and $F(z)$. From equation (3)

$$
\phi \equiv \arg s_{0}=\arg \left[-\frac{\cos \alpha}{2}+i\left(\frac{\sin \alpha}{2}-\frac{1}{4 \sin \alpha}\right)\right]=-2 \alpha+\frac{3 \pi}{2},
$$

so that as $\alpha$ runs through the open interval $(0, \pi)$ the angle $\phi$ runs through the open interval $(-\pi / 2,3 \pi / 2)$ in the reverse order. The endpoints $\phi=-\pi / 2$ or $3 \pi / 2$ correspond to the case $a=0, B<0$ and this case cannot occur. Thus we can always find $\alpha$ so that $\arg x_{0}=\arg (a+B i)$ and then we can find a $t>0$ so that $s_{0}=t(a+B i)$.

Once this selection is made, then $B(z)=G(t f(z))$ satisfies the conditions of Schwarz's lemma. Since $B^{\prime}(0)=G^{\prime}(0) t f^{\prime}(0)=t$, we see that $t<1$ and $t=1$ if and only if $f(z) \equiv F(z)$. Thus $a+B i$ must lie outside the domain bounded by the curve described by $s_{0}$. Setting $\rho=\left|s_{0}\right|$, we have from (3)

$$
\rho=\frac{1}{4 \sin \alpha}=\frac{1}{4 \sin (3 \pi / 4-\phi / 2)} .
$$

Thus (16) gives the equation in polar coordinates of the curve described by $s_{0}$.

A simple argument shows that equation (16) gives the boundary of the covered domain $\mathscr{D}$ if the omitted point is in the upper half-plane, but if the omitted point is in the lower half-plane we must replace $F(z)$ by $\overline{F(\bar{z})}$. We have proved 
THEOREM 1. Let $\mathscr{D}$ be the largest domain contained in $f(E)$ for every function $f$ in the family $C D$. Then $\mathscr{D}$ is bounded in the upper half-plane by the curve (16) for $0<\phi<\pi$, and $\mathscr{D}$ is bounded in the lower half-plane by the reflection of this curve in the real axis.

ADDED IN PROOF. After this paper was accepted for publication, we learned that Theorem 1 was proved earlier by M. O. Reade and E. J. Zlotkiewicz [On univalent functions with two preassigned values, Proc. Amer. Math. Soc. 30 (1971), 539-554]. However, our proof is simpler than theirs. They describe the domain that is always covered, by the inequality $8|w|(|w|+|\operatorname{Im} w|)<1$.

\section{REFERENCES}

1. W. Hengartner and G. Schober, On schlicht mappings to domains convex in one direction, Comment. Math. Helv. 45 (1970), 303-314.

2. A remark on level curves for domains convex in one direction, Applicable Anal. 3 (1973), 101-106.

3. M. S. Robertson, Analytic functions star-like in one direction, Amer. J. Math. 58 (1936), 465-472.

4. W. C. Royster and Michael Ziegler, Univalent functions convex in one direction, Publ. Math. Debrecen 23 (1976), 339-345.

Department of Mathematics, University of South Florida, Tampa, Florida 33620 